\title{
Dalla produzione automatizzata agli ambienti tecnologicamente densi: la dimensione sociomateriale dell'agire organizzativo
}

\author{
di Attila Bruni e Laura Lucia Parolin
}

La produzione automatizzata dei prodotti e dei servizi, quale sostituzione tecnica del lavoro umano e dei processi organizzativi, è da sempre al centro dell'interesse di diverse discipline che partecipano al campo degli studi organizzativi: sociologia, economia, ingegneria, scienze dell'informazione, ergonomia e design. Allo stesso tempo, la densità tecnologica degli ambienti di lavoro contemporanei porta a una sempre maggiore distribuzione e frammentazione delle attività tra attori umani e non umani e pone domande nuove, che riguardano sia i concetti e le categorie analitiche, sia le metodologie di analisi e di design dei sistemi sociotecnici.

Sebbene le discipline organizzative siano da sempre interessate alla tecnologia, la riflessione accademica sul tema del rapporto tecnologia e organizzazione appare negli ultimi anni notevolmente cambiata. Analizzando la letteratura organizzativa e manageriale degli ultimi anni, Orlikowski e Scott (2008) evidenziano come, malgrado la presenza sempre più pervasiva nelle pratiche organizzative, il tema della tecnologia sia sorprendentemente quasi scomparso dal dibattito. Da un lato si è affievolito l'interesse stesso verso le "determinanti tecnologiche" della struttura organizzativa (Woodward, 1965; Perrow, 1984), dall’altro è diminuita la preoccupazione circa la sostituzione del lavoro umano su base tecnologica, visto lo slittamento delle attività dal piano fisico e quello cognitivo.

Eppure, tecnologie e infrastrutture della comunicazione sono divenute sempre più presenti nelle pratiche e negli spazi organizzativi, rendendo peraltro sempre meno evidente e scontata la distinzione tra lavoro umano e lavoro delle macchine. In altre parole, Come nota Lucy Suchman (2002) nell'introduzione alla seconda edizione del suo rinomato testo Plans and Situated Action (Suchman, 1987), se negli anni Ottanta il focus della riflessione riguardava il dove inserire una linea di demarcazione tra umani e 
non-umani, negli anni Duemila la questione diventa come inserire una linea di demarcazione tra azione umana e azione tecnologica. Nel mondo occidentale attuale, la socialità delle macchine è infatti ormai un "dato per scontato", in quanto i software interattivi assumono sempre più la forma di 2"knowbots", macchine sapienti che automatizzano la conoscenza allo stesso modo in cui i robot dovevano automatizzare la produzione materiale. Gli oggetti sono oggi diventati elementi costitutivi del sapere (quali incubatori di conoscenza) e delle relazioni sociali (quali risorse identitarie e di connettività diffusa), fondando così ciò che Knorr-Cetina (1997:12) definisce come object-centered sociality. Il problema, allora, non è più se le macchine possiedano capacità d'azione, bensì come definire l'azione, umana o nonumana che sia (Lynch e Collins, 1998).

Proprio la capacità di leggere l'organizzazione come risultato emergente dell'intra-azione tra umano, sociale e tecnico, diviene la caratteristica della corrente di studi che Orlikowski e Scott (2008) identificano come uno dei filoni maggiormente promettenti per i futuri studi organizzativi in materia di tecnologia. Si tratta di una corrente che trova le sue radici teoriche negli studi sociali sulla scienza e la tecnologia (Science and Technology Studies - STS) e la cui rilevanza per gli studi organizzativi e dei processi economici e del lavoro è già stata variamente dibattuta e accreditata (Law, 1994; Gherardi e Lippi, 2000; Knorr Cetina e Preda, 2005; Czarniawska e Hernes, 2005; Pinch e Swedberg, 2008).

Lo spostamento di prospettiva che proponiamo in questo special issue riflette dunque una più ampia evoluzione del dibatto in materia di tecnologie, non più intese come entità a sé stanti che impattano sulla dimensione sociale e organizzativa, ma parte integrante e costitutiva degli ambienti organizzativi e dei corsi di azione che in essi prendono forma.

\section{La rivista e il dibattito}

La rivista Studi Organizzativi vanta una lunga tradizione di contribuiti sul tema delle tecnologie nelle organizzazioni e nel lavoro. Con il seminario: «Dalla produzione automatizzata agli ambienti tecnologicamente densi. Giornata di Studi su tecnologia e organizzazione», tenutosi al dipartimento di Sociologia dell’Università Milano Bicocca l'8 Ottobre 2013, abbiamo voluto aprire uno spazio di riflessione e dibattito circa lo stato dell'arte negli studi organizzativi a proposito dell’intreccio tra tecnologie, lavoro, e organizzazione. La Giornata di Studi, anticipata da un call for paper ed un processo di review interna, ha così riunito numerose studiose e studiosi im- 
pegnati nella comprensione del lavoro in ambienti e situazioni organizzative a forte caratterizzazione tecnologica e si è articolato in tre distinte sessioni:

Piattaforme tecnologiche e modelli organizzativi (coordinata da Fabrizio Panozzo);

Tecnologie e innovazione (coordinata da Ivana Lo Nigro);

Tecnologie, saperi e pratiche organizzative (coordinata da Attila Bruni).

Relatori e relatrici hanno presentato ricerche empiriche svolte in contesti organizzativi tra loro eterogenei (imprese, cantieri edili, laboratori scientifici, uffici della pubblica amministrazione, servizi socio-sanitari), per quanto dalle discussioni scaturire sia apparso evidente come la quotidianità di realtà organizzative apparentemente distanti sia ormai sempre e comunque caratterizzata dalla presenza di una varietà di artefatti tecnologici che supportano, permettono e influenzano le attività stesse.

Il presente numero speciale intende pertanto riprendere la riflessione avviata nel corso del seminario al fine di articolare un dibattito più ampio sulle forme di co-partecipazione di umani e tecnologie nell'agire organizzativo. In particolare, in questa sede ci chiediamo: come le riflessioni sull'automazione e sull'introduzione delle tecnologie ICT nei luoghi di lavoro possono essere utili a comprendere le caratteristiche dell'agire organizzativo? Che tipo di elementi illumina (o oscura) un focus puntato sui processi di automazione? È ancora utile una suddivisione tra lavoro umano e non umano? Come comprendere all'unisono il lavoro di individui, gruppi e tecnologie nelle organizzazioni contemporanee? Quali sono gli strumenti analitici e le professionalità necessarie alla progettazione di ambienti organizzativi a forte integrazione sociotecnica?

\section{Le riflessioni sul tema}

Nel tentativo di dare risposta a queste domande, il presente numero affianca ad articoli provenienti dalla ricerca empirica alcuni contributi di riflessione teorica che si interrogano sull'intreccio tra lavoro, tecnologia e organizzazione a partire da prospettive legate a diversi ambiti disciplinari (psicologia sociale, sistemi informativi, sociologia dell'organizzazione). Il filo conduttore, come vedremo, è un modo di concepire la tecnologia e l'automazione non in riferimento alla configurazione e affidabilità tecnica, bensì in relazione al contesto organizzativo, alle esigenze degli utenti e alle pratiche di lavoro quotidiane. Il tema centrale è la necessità di adottare un 
approccio relazionale (Suchman, et al., 1999), che non legga le tecnologie come entità separate dagli utilizzatori e dalle pratiche d'uso, bensì le consideri nelle reciproche interazioni che con essi vengono a crearsi (o a mancare).

Proprio in virtù di un approccio che sembra riecheggiare la raccomandazione sociotecnica circa la necessità di progettazione congiunta della componente tecnologica e sociale dell'organizzazione (Emery e Trist, 1981), Federico Butera sottolinea la necessità di governare i processi di automazione attraverso forme di progettazione integrata. A partire da una disamina della storia del termine "automazione” negli studi organizzativi e di management, l'articolo di Butera introduce ad una riflessione sulla relazione tra tecnologia e organizzazione. Sebbene l'interesse verso le tecnologie meccaniche nel lavoro abbia una storia antica, è solo con la diffusione della produzione di massa che si è iniziato a pensare di poter realizzare sistemi di produzione del tutto automatizzati. Storicamente, gli studiosi che si sono soffermati sul tema dell'automazione hanno alternato a scenari apocalittici, visioni utopiche preconizzanti mondi nei quali gli esseri umani svolgono unicamente attività di supervisione e controllo del lavoro delle macchine. La letteratura che si è concentrata sugli effetti sociali dell'automazione ha tuttavia perlopiù mostrato come questa produca non tanto la sostituzione delle macchine agli umani o la "liberazione" degli umani dal lavoro, quanto uno spostamento (se non un vero e proprio slittamento) delle attività e delle competenze richieste al lavoro umano (Cedefop, 2010).

Non sfuggono all'autore le criticità connesse a una proliferazione di sistemi altamente automatizzati. In particolare attraverso la mediazione tecnologica si allontana il lavoro umano dall'attività e dal sensemaking organizzativo a essa connessa (Weick, 1979), rischiando di togliere all'operatore la consapevolezza delle situazioni (Weick, 1990). L'automazione implica dunque qualcosa di più complesso del semplice spostamento di attività umane di tipo operativo alle macchine perché è al contempo un processo di cambiamento sociale e tecnico che coinvolge l'intera organizzazione e implica una diversa distribuzione della conoscenza tra umani e macchine. Secondo Butera può quindi esistere un margine di manovra per il miglioramento organizzativo se nella progettazione "integrata” di processi, tecnologia, ruoli e cultura organizzativa si mantiene al centro l'attore umano, con le esperienze e le conoscenze che gli sono proprie.

La proposta di Butera si connota per l'assunzione di responsabilità verso una progettazione integrata in grado di assicurare il design dei ruoli, dei gruppi e delle attività in modo congiunto alla progettazione tecnica di arte- 
fatti e sistemi di supporto delle attività lavorative. Il metodo di lavoro proposto è un coinvolgimento corale dell'organizzazione per permettere agli operatori, così come ai responsabili delle unità organizzative, di indicare e discutere esigenze, priorità e caratteristiche delle attività insieme al team interdisciplinare incaricato dell'intervento di progettazione (Butera, 2009).

La consapevolezza che una progettazione dell'automazione lontana dagli ambienti organizzativi e che attribuisce all'operatore umano un ruolo residuale sia facilmente destinata a fallire, si è peraltro sviluppata anche all'interno del campo della Human-Computer Interaction e di progettazione dei sistemi informativi, da cui proviene la riflessione con cui Maurizio Teli, Vincenzo D'Andrea e Antonella De Angeli contribuiscono a questo numero speciale. Gli autori ci introducono a un orientamento sempre più diffuso nell'ambito dei recenti studi di Participatory Design e di Human Computer Interaction, che recupera una visione eticopolitica della progettazione. Riprendendo la suddivisione tra automazione e informatizzazione proposta da Zuboff (1988), gli autori propongono una riflessione intorno al tema del valore d'uso della tecnologia stessa. A muovere tali considerazioni è la critica a una visione della tecnologia come portatrice intrinseca di valori positivi: «Valore per chi?», è la domanda dalla quale parte la riflessione degli autori.

Il contributo di Teli e colleghi ci permette inoltre di focalizzare l'attenzione sull'evoluzione del concetto di usabilità sviluppata all'interno della Human Computer Interaction. L'usabilità non si riferisce esclusivamente alle caratteristiche tecniche dell'artefatto ma deve includere i soggetti nello specifico momento della relazione: deve essere cioè ambientata in situazione (e non in setting sperimentali). Come sostengono gli autori, l'efficacia del flusso di azione che coinvolge contemporaneamente esseri umani e macchine è in stretta connessione con l'allineamento tra le affordance (Gibson, 1979) della macchina, le occasioni e i vincoli che il contesto suggerisce, e gli obiettivi/interessi che guidano l'azione umana. Il concetto di usabilità dunque esce dal dominio della tecnica per includere l'esperienza e le pratiche d'uso. In tale ambito le riflessioni di Zuboff su come gli stili di progettazione di tecnologie possano essere orientati al controllo organizzativo invece che allo sviluppo delle competenze degli utenti, assumono un interesse rinnovato. Come la progettazione può supportare dinamiche di democratizzazione del lavoro e sviluppare le competenze di chi le usa?

La risposta a questa domanda sollecita l'adozione di una prospettiva capace di guardare alle tecnologie come pratiche sociali (Suchman et al., 1999). Le tecnologie sono cioè inseparabili dal loro uso e questo è sempre immerso in un campo di relazioni con gli altri strumenti e altre pratiche 
(organizzative e di lavoro, nel nostro caso). Se la letteratura che si è interrogata sulle conseguenze sociali dell'automazione ha evidenziato uno spostamento del lavoro verso attività sempre più di controllo, cognitive e relazionali (Cedefop, 2010), studiando i tecnici riparatori di macchine fotocopiatrici, Julian Orr (1996) illustra la conoscenza pratica necessaria alla manutenzione della relazione macchina/cliente. L'azione esperta dei tecnici considera infatti tecnologie e utilizzatori come parti di un unico sistema (il corretto funzionamento di una macchina dipende anche dai comportamenti dei suoi utenti), all'interno del quale, quindi, talvolta sono le tecnologie a essere difettose, ma altre sono gli utenti a relazionarsi in modo scorretto con la macchina e ad abbisognare di un "aggiustamento". Umani e tecnologie abitano, e al tempo stesso strutturano, gli ambienti organizzativi, come messo in evidenza anche da ricerche svolte nelle sale di controllo di metropolitane (Luff et al., 2000), aeroporti (Suchman, 1997), ambulanze (Heath et al., 2000), autostrade (Bruni e Modè, 2011) e, più in generale, in tutte quelle situazioni in cui il coordinamento a distanza è permesso dall'impiego di articolate infrastrutture tecnologiche (Engeström e Middleton, 1996).

Sulla relazione tra strumenti tecnici e lavoro Francesca Alby presenta il punto di vista della psicologia culturale. Mentre la psicologia cognitiva non si è mai interessata molto agli artefatti perché ritenuti parte del contesto e dunque ininfluenti sui processi mentali (Mantovani, 2004), la psicologia culturale ritiene che i processi psicologici siano mediati dagli artefatti culturali e sempre costruiti nell'interazione sociale (Vygotskij, 1934). Secondo tale ottica gli artefatti sono mediatori che, mentre permettono di realizzare azioni pratiche, strutturano la mente e le sue attività. Come argomenta Alby, abbiamo imparato l'attività della scrittura come sistema integrato tra l'attività manuale (che ci rende in grado di usare una penna) la modalità narrativa con cui pensare cosa scrivere. Le funzioni psichiche della percezione sono legate alla partecipazione a contesti quotidiani e alla competenza nel manipolare artefatti e sistemi di mediazione che abitano, e al tempo stesso strutturano, tali contesti. Gli attori umani non si limitano a conoscere il mondo o ad agire in conformità a norme sociali, ma attivamente costruiscono la loro realtà quotidiana contribuendo al mantenimento e alla variazione delle norme di riferimento.

Il contributo di Alby ci introduce così ad un approccio al conoscere (più che alla cognizione) quale attività (invece che come corpo di conoscenze), ed in particolare quale attività situata, cioè emergente dal contesto di sua produzione ed ancorata dai (e nei) supporti materiali di tale ambiente. Da meri contenitori di attività, i luoghi di lavoro divengono così contesti 
di attività: essi ci aiutano a ricordare, ci consentono di fare alcune cose e di non farne altre, ci interpellano con segnalazioni visive o uditive per sollecitare un nostro intervento, ci interrogano con programmi che ci aiutano a diagnosticare eventuali guasti e ci suggeriscono come intervenire.

\section{Il costrutto “ambienti tecnologicamente densi”}

Questa parentesi sulla natura dell'azione e della cognizione che la psicologia culturale e altre correnti delle scienze umane e sociali propongono oggi, ci permette di leggere il rapporto tra tecnologia, lavoro e organizzazione in modo più articolato di quanto non sia stato osservato tradizionalmente negli studi sull' automazione. L'espressione "ambienti tecnologicamente densi” (Bruni, 2005; Bruni et al., 2013) riflette questo cambio di prospettiva e cerca di attirare l'attenzione su come nelle organizzazioni contemporanee si costruiscano (e agiscano) attraverso articolate pratiche sociomateriali, che affiancano umani e non umani e danno forma alle attività. In tal senso, possiamo assimilare agli ambienti tecnologicamente densi tutte quelle situazioni organizzative in cui:

- lavorare implica complesse pratiche sociomateriali ed uno specifico know-how tecnologico;

- umani e tecnologie lavorano 'insieme';

- l'interazione è resa possibile dalle tecnologie e spazio e tempo vengono riconfigurati sulla base di tali interazioni (e tecnologie).

Più che quale "concetto definitorio" (che dica quindi cosa guardare), preferiamo intendere il costrutto di TDEs quale 'concetto sensibilizzante' (Blumer, 1969) capace di indicare una direzione in cui cercare. Pensiamo infatti che la densità tecnologica che caratterizza alcuni ambienti organizzativi non sia da assumere quale punto di partenza, ma vada ricercata quale proprietà emergente degli ambienti considerati. In altre parole, la densità tecnologica di un ambiente organizzativo non dipende tanto dalla presenza di diverse tecnologie, quanto dalla qualità delle relazioni che tra esse s'instaurano e dal tipo di interconnessioni che vengono a crearsi tra il loro uso e le pratiche organizzative e di lavoro quotidiano (Bruni et al., 2013). Ciò vuol dire assumere una prospettiva relazionale e non determinista, che non assegni agli artefatti tecnologici un ruolo predefinito e che sia invece interessata agli usi situati di questi ultimi, a seconda della rilevanza che per 
gli attori essi rivestono nello svolgersi concreto della quotidianità lavorativa.

Pensare alle organizzazioni quali TDEs è quindi un'operazione metaforica volta ad accentuare l'intima relazione che si instaura tra contesto lavorativo, artefatti tecnologici e strutturazione delle attività. Preferiamo tuttavia parlare di "ambiente" (invece che di "contesto") per rimanere fedeli a una concezione ecologica dell'azione (Ingold, 2000) che sottolinei le proprietà "generative" (e non solo normative) dell'azione nel mondo (Schutz, 1974). Nel momento in cui la tecnologia cessa di essere un semplice elemento di supporto e diventa una vera e propria conditio sine qua non per il concretizzarsi del lavoro e dell'azione dei partecipanti, l'ambiente non rappresenta più il contesto d'uso che assorbirà la tecnologia, ma, al limite, il contesto d'uso reso possibile dalla tecnologia ed all'interno del quale si attua il coordinamento delle pratiche.

\section{Fare ricerca con i TDE}

Cosa ci fanno vedere dei TDE (e dei processi legati alla sociomaterialità dell'agire organizzativo) gli articoli presenti in questo numero speciale di Studi Organizzativi?

Nel loro complesso, tutti puntano l'attenzione sull'intersezione tra tecnologie e organizzazione quale processo aperto e mai concluso, seppure costantemente alla ricerca di un qualche tipo di 'chiusura' e conclusione. Tre articoli, in particolare, prendono le mosse da ricerche svolte in ambito sanitario e/o in organizzazioni ospedaliere. Gli ambienti ospedalieri costituiscono un classico esempio di "ambiente tecnologicamente denso", dove oggetti tecnici e saperi tecnoscientifici partecipano in maniera sostanziale alle pratiche, alle relazioni e all'organizzazione del lavoro quotidiano (Casper e Berg, 1995; Bruni, 2005; Bruni, 2010). La diffusione delle tecnologie dell'informazione e della comunicazione nel mondo della sanità, inoltre, ha avuto un notevole impatto sull'organizzazione dei sistemi sanitari, sulla pratica professionale e sull'esperienza che i pazienti hanno della malattia e della cura (Danholt et al., 2013), come indicato anche dalle sempre più frequenti esperienze (e tentativi) di gestione 'a distanza' di alcune patologie (Mort et al., 2003; Gherardi e Strati, 2004; Parolin, 2011).

Più nel dettaglio, ciascun contributo si sofferma su un tratto caratteristico dei TDE, ovvero su una specifica pratica che all'interno di questi prende forma e ne specifica le qualità. Nell'articolo di Piras e Zanutto, e in quello di Crabu, tale pratica coincide con quello che in termini più generali 
potremmo identificare come lavoro invisibile (Star e Strauss, 1998) e che tuttavia, proprio per via della sua componente 'materiale' (ovvero dell'uso di tecnologie e oggetti che esso sottintende), nei TDE diviene manifesto.

Nel caso del reparto ospedaliero e della cartella clinica informatizzata al centro dello studio di Enrico Maria Piras e Alberto Zanutto, ad esempio, vediamo la rilevanza che assume il "lavoro di congiunzione" svolto quotidianamente dal personale infermieristico al fine di assicurare l'interoperabilità tra le componenti umane e tecniche dell'organizzazione e all'interno delle stesse. Se, infatti, il fine principale del lavoro di congiunzione è garantire che le informazioni prodotte all'interno del settore sanitario-ospedaliero siano sempre disponibili e di facile accesso per i medici, è importante notare come ciò implichi assicurare i collegamenti tra umani e tecnologie, ma anche quelli tra tecnologie e tecnologie e/o tra umani e umani. Infatti (sottolineano gli autori), vi sono casi in cui, per quanto sistemi informativi diversi siano stati resi interoperabili da un punto di vista tecnico, il lavoro di congiunzione si rende necessario a fronte (ad esempio) di un malfunzionamento; in altri casi, il lavoro di congiunzione è ciò che permette ad artefatti tecnologici caduti in disuso rispetto al loro utilizzo "tradizionale" di assolvere a nuovi compiti; in altri ancora, è ciò che rende intercambiabili alcuni strumenti; ed in altri casi ancora, è il lavoro di congiunzione a far sì che informazioni e conoscenze viaggino tra umani. Il lavoro di congiunzione è ciò che tiene insieme attività “analogiche” (quali prendere appunti o ricopiare note e biglietti temporanei) e uso di diversi supporti digitali, a dimostrazione di come in ambienti ad alta densità tecnologica le 'nuove tecnologie' non spazzino via quelle vecchie, ma vi si affianchino, dando vita a una sorta di 'stratificazione tecnologica' che richiede come (nuova) abilità il sapersi destreggiare tra materiali e strumenti di fattura differente. A questo proposito, notano ulteriormente gli autori, il lavoro di congiunzione contribuisce a modificare il contenuto della professione, ridefinendone i confini e riorientando lo sviluppo delle competenze organizzative.

Nel caso di Stefano Crabu, avente ad oggetto la creazione, l'utilizzo e la contestualizzazione di un protocollo nell'ambito della biologia molecolare, il lavoro invisibile assume invece caratteristiche "infrastrutturanti". Le descrizioni etnografiche che l'autore propone, trasportano il lettore nel mondo quotidiano di un importante laboratorio di ricerca, mostrando come dietro un "protocollo pronto all'uso", si celino routine, saperi e conoscenze, "infrastrutturali” perché date per scontate e ritenute patrimonio collettivo della comunità scientifica in cui il protocollo andrà ad operare. Il "lavoro infrastrutturante” rimanda quindi a tutte quelle attività (quanto mai comuni 
nelle organizzazioni contemporanee) finalizzate a cristallizzare testi, discorsi orali, saperi e conoscenze situate all'interno di linee guida, best practice e/o protocolli. L'articolo, in continuità con una letteratura STS e lungi da una descrizione romantica del "fare scienza", permette di osservare come la definizione di standard procedurali, lungi dallo svolgersi in termini astratti e oggettivi, si appoggi largamente ad attività discorsive e di uso situato di oggetti e tecnologie. Così facendo, l'articolo restituisce un'idea del fare scienza quale attività segnata oggi più che mai da un insieme di processi tipicamente organizzativi, che vanno dall'apprendimento delle giuste “regole dell'attenzione” (March, 1988), alla gestione (e all'aggiornamento) delle diverse conoscenze e expertise che l'uso di tecnologie e protocolli incorpora (Viteritti, 2012), sino al lavoro “a contatto di gomito" (Hughes, 1958), che permette che ognuno conservi le proprie responsabilità mentre lavora insieme ad altri. Da notare che, a differenza dell'epoca in cui scriveva Hughes, oggi questi "altri” non sono solo attori umani, ma anche tecnologie e testi.

A questo proposito, può essere utile ricordare che dare un agency ad oggetti e "materiali” non significa scadere in forme di animismo, bensì volersi confrontare con la materialità dell'agire sociale (Knorr Cetina, 1997) e dunque con la "naturalezza" che gli oggetti tecnici acquistano agli occhi degli attori (Suchman, 2007). Negli ambienti che incontrano, tecnologie e oggetti "fanno" e "fanno fare", come insegnano gli studi sociali su scienza e tecnologia (Mattozzi, 2005), e costruiscono le organizzazioni (e il sociale tout court) come fenomeni materialmente eterogenei (Law, 1994), assemblati a partire da oggetti tecnici, corpi, testi, macchine, architetture e così via (Latour, 2005).

La più recente letteratura STS è solita fare riferimento al cognitivismo ecologico di William Gibson per rendere conto della natura relazionale ed al contempo materiale degli oggetti. Gibson (1979) propone di leggere gli oggetti in termini di affordance, come se la materialità del mondo costituisse un "invito a", una "occasione per", un repertorio di azioni (ad esempio, un coltello ha l'affordance del taglio, ma anche della minaccia). Il fatto che gli oggetti abbiano diverse affordance significa quindi che essi possono acquisire differenti identità a seconda dell'attività che li costruisce a livello pratico, ma sempre e comunque a partire da alcuni fattori materiali (Harré, 2002). In tal senso, è importante notare come le affordance richiamino l'attenzione degli attori su alcune delle possibilità offerte dall'ambiente (una porta "invita" al passaggio), ma al tempo stesso dipendano dagli interessi e dalla capacità di azione di questi ultimi (non è detto che gli attori ricer- 
chino l'affordance del passaggio, né che l'apertura della porta sia immediatamente accessibile a chiunque).

In riferimento agli ambienti organizzativi (ma non solo), ciò significa che il ruolo più o meno 'attivo' che oggetti e tecnologie possono rivestire non è dovuto alle loro proprietà tecniche, bensì al tipo di relazione che, nella pratica, li lega a diversi soggetti ed all'abilità di questi ultimi nel trasformare gli elementi presenti nell'ambiente fisico in risorse utili all'azione. Non a caso, ricerche condotte in ambienti organizzativi tra loro anche molto diversi (dai call center ai mercati finanziari, alle sale di coordinamento degli aeroporti, delle ambulanze e della metropolitane), ma accomunate da un certo tipo di attenzione per gli usi situati della tecnologia (Suchman et al., 1999; Heath e Luff, 2000; Beunza e Stark, 2003), mostrano come il lavoro possa essere interpretato alla stregua di una performance, che prende forma a partire da una coreografia improvvisata (Whalen et al., 2002) dei diversi elementi che compongono la scena. Per quanto "coreografia improvvisata" 1 possa apparire una metafora contraddittoria (l'improvvisazione rimanda ad un'azione estemporanea, la coreografia ad una sequenza stabilita di azioni), l'immagine serve ad attirare l'attenzione proprio sulla compresenza dei due aspetti, ossia su come, per quanto tecnologie e oggetti "strutturino" la scena, il procedere del lavoro sia assicurato a partire da un uso potenzialmente sempre nuovo degli elementi a disposizione.

Ecco che allora pensare agli ambienti organizzativi contemporanei quali ambienti tecnologicamente densi permette di rovesciare il problema circa cosa distingua il lavoro umano dal lavoro delle macchine e indagare invece cosa implichi e quali competenze richieda in termini organizzativi abitare ambienti e pratiche che mobilitano l'azione congiunta di umani e tecnologie (Lynch e Collins, 1998). È infatti a partire da tale ribaltamento della prospettiva che l'articolo di Roberto Cibin, Lorenzo Ruzzene e Francesca Gleria ci introduce a una questione (gli open data) e ad un ambiente organizzativo (la pubblica amministrazione) diversi, ma che chiamano in causa processi e problemi altrettanto centrali per la comprensione delle "forme di vita tecnologica” (Lash, 2000) che caratterizzano le organizzazioni oggi. Il tema della gestione del patrimonio informativo delle PA e, più in generale, della possibilità per i cittadini di accedere a (e utilizzare) i dati resi pubbli-

\footnotetext{
${ }^{1}$ Espressione coniata da Whalen e colleghi (2002) in riferimento all'abilità degli operatori dei call-center di giostrare i diversi elementi coinvolti all'interno di una telefonata 'auditivamente competente': il corpo dell'operatore, il turno di parola, il software su cui è basato il sistema e gli oggetti di lavoro di uso quotidiano.
} 
ci, ha delle implicazioni tanto a livello organizzativo, quanto giuridico e politologico (Gurstein, 2011), in quanto implica passare da una concezione "proprietaria” del dato ad una "comunitaria”. Ma da un punto di vista organizzativo, notano gli autori in riferimento al progetto Open Data in Trentino (avviato dalla Provincia Autonoma di Trento al fine di aprire al pubblico il patrimonio informativo presente negli archivi delle diverse strutture provinciali e sviluppare servizi per e con i cittadini), la questione principale risiede nel modo in cui gli open data riconfigurano i confini della pubblica amministrazione e le reti istituzionalizzate che fino a questo momento avevano caratterizzato la gestione delle informazioni da parte delle strutture provinciali. La ricerca condotta (ancora una volta, con l'ausilio di tecniche etnografiche, punto sul quale torneremo tra breve), evidenzia infatti la costruzione di una nuova rete d'azione, che travalica i confini istituzionali e che mobilita un network di attori eterogenei: organizzazioni del sistema provinciale, gruppi di ricerca, imprese, sistemi informativi, norme, cittadini e infrastrutture tecnologiche. Come è facile immaginare, ognuno di questi attori nutre interessi diversi: le strutture provinciali devono adempiere ad obblighi di legge e vedono nella pubblicazione dei dati la possibilità di rendere più efficiente la propria organizzazione e favorire la crescita del territorio; i cittadini aspirano a processi di partecipazione diffusa e di coprogettazione dei servizi; le imprese ricercano nuove partnership e "capitali informativi" da gestire per conto di terzi. Per quanto il progetto versi ancora nelle sue fasi iniziali (e sia dunque prematuro trarre conclusioni certe), gli autori intravedono uno scenario in cui il processo stesso di apertura del dato fa sì che l'ambiente tecnologicamente denso si espanda oltre i confini organizzativi della pubblica amministrazione stessa, per coinvolgere le pratiche di cittadini, associazioni, imprese e/o altri enti. Al contempo, ciò riverbera anche sulle pratiche organizzative e lavorative dei funzionari dell'ente pubblico coinvolto nel progetto, che dovrà acquisire familiarità con nuovi aspetti legali, tecnici, di processo, nonché di visibilità e controllo (ora anche pubblico) del proprio operato.

Peraltro, è da notare come la ricerca stessa abbia contribuito ad "aprire" i confini della PA: il gruppo degli autori è composto da un funzionario provinciale esperto e due collaboratori messi a disposizione da due organizzazioni afferenti al sistema pubblico. Fin dall'inizio del progetto, gli autori (nella loro veste lavorativa) hanno proceduto alla definizione e al monitoraggio, in collaborazione con gli altri attori del progetto, delle azioni da intraprendere per avviare il percorso di sensibilizzazione e formazione sul tema degli open data all'interno delle strutture provinciali e per portare i diversi uffici ad iniziare a rendere pubblici i propri dati. 
Siamo quindi di fronte a uno di quei casi in cui, per quanto teoricamente orientata da una lettura prossimale della relazione tra tecnologia e organizzazione (Cooper e Law, 1995), la ricerca non può dirsi "pura”; dove i ricercatori sono al contempo attori dei processi studiati; e dove l'etnografia diviene parte integrante delle azioni di innesco del cambiamento organizzativo. L'etnografia, peraltro, ha conquistato una posizione di sempre maggiore rilievo nelle pratiche di ricerca degli studi organizzativi, come segnalato anche dalla comparsa (nel 2012) del Journal of Organizational Ethnography. È innegabile che parte del rilievo dato oggi all'etnografia abbia origine da un interesse prevalentemente applicativo (Bruni e Gherardi, 2007): lo sviluppo delle tecnologie della comunicazione e dell'informazione, la crescente complessità degli ambienti lavorativi, la rilevanza dei network e delle pratiche di networking, hanno infatti posto al centro del dibattito il lavoro invisibile a cui sono chiamati tanto gli utenti delle tecnologie, quanto gli attori organizzativi, assieme a tutto il variegato mondo di pratiche e conoscenze tacite che sembrano accompagnare la cooperazione, il coordinamento e, più in generale, qualunque forma di agire “competente" ed "esperto". L'etnografia consente infatti di analizzare, attraverso l'osservazione e la narrazione, il "farsi" ed il "fare" di diverse pratiche organizzative, ponendo in evidenza dettagli che contribuiscono ad ampliare i punti di vista sulle problematiche del contesto oggetto di studio. L'etnografia è, per chi vi s'immerge, un processo di apprendimento delle pratiche indagate per come queste vengono vissute, narrate e interpretate dagli attori del campo (Piccardo e Benozzo, 1996; Bruni, 2003). Nell'etnografia conta quindi il gioco di interazioni che costruisce un 'normale' senso di realtà, il ruolo degli oggetti e degli artefatti, il corpo e le emozioni degli attori indagati, ma anche dell'etnografo (Bruni e Viteritti, 2011).

Come, dunque, l'etnografia organizzativa può aiutarci nella comprensione dei TDE e nell'innescare cambiamento organizzativo?

Con questo tema si confronta l'articolo di Barbara Mellini, Sabina Giorgi e Alessandra Talamo, dove spazi e artefatti organizzativi divengono parte integrante della traiettoria seguita dalla ricercatrice al fine di raggiungere il cuore delle pratiche dell’Unità Operativa Complessa (U.O.C) di un ospedale romano. L'uso dell'etnografia in riferimento allo studio delle comunità di pratiche rappresenta un classico sin dallo studio di Lave e Wenger (1991); meno battuto è stato invece l'impiego di tecniche etnografiche in relazione a una "comunità di oggetti” (Bruni, 2005; 2011). Per altrettanto, se l'identità del ricercatore e dell'accesso al campo sono temi dibattuti da un punto di vista metodologico nell'etnografia contemporanea (Silver- 
man, 1997; Cardano, 2013), una riflessione a proposito di come sia possibile rintracciare delle tracce significative delle pratiche organizzative già nelle attribuzioni identitarie che gli attori del campo rivolgono verso l'etnografo e nella traiettoria di accesso al campo da quest'ultimo seguita rimane ancora limitata (Bruni, 2006, 2007).

Ecco che allora le autrici eleggono a oggetto privilegiato del loro resoconto etnografico proprio le diverse tappe della traiettoria d'accesso all'organizzazione seguita da colei che ha condotto il lavoro sul campo. Tappe che, sulla scorta della metafora dell'organizzazione quale teatro (Morgan, 1986; Goffman, 1959), divengono "scene”, “atti”, “rappresentazioni" in cui gli attori organizzativi sono chiamati a performare il proprio ruolo professionale in relazione a una situazione inedita, quale quella in cui un 'estraneo' chieda di poter osservare la vita quotidiana dell'organizzazione. Entro tale performance definitoria, notano le autrici, l'uso strumentale da parte degli attori di alcuni artefatti organizzativi, tra cui gli spazi fisici di attraversamento e gli "artefatti di soglia" (Zucchermaglio, Alby, Fatigante, Saglietti, 2013), restituisce la 'materialità' di alcune logiche e pratiche organizzative, divenendo un altro degli elementi che consentono all'etnografa di avvicinarsi a (e comprendere) il contesto indagato. La ricercatrice stessa, con la sua presenza, diviene un attivatore di dinamiche relazionali e interprofessionali generalmente implicite, sino a divenire a sua volta un "artefatto di soglia" conteso tra direzione e personale infermieristico nel tentativo di far valere le rispettive esigenze.

Focalizzare l'attenzione sugli artefatti che partecipano alle diverse "scene" che permettono l'accesso al campo, consente così di vedere dove si va a creare e ricreare l'organizzazione: questa si annida proprio nei dettagli del coordinamento (riuscito o mancato che sia) tra le componenti sociomateriali del più complesso sistema sanitario ed è tenuta insieme dagli artefatti che connettono pratiche, attori e logiche eterogenee. L'articolo viene così a rappresentare un interessante esempio di quello che può essere il contributo dell'etnografia allo studio dei TDE e nell'attivare processi di cambiamento all'interno di specifici setting organizzativi. Eleggere gli artefatti organizzativi (e l'uso e il non-uso che ne viene fatto all'interno di contesti d'azione situata) a "s-oggetti" dell'osservazione (Bruni, 2005) e interpretarli per quelle che sono le loro caratteristiche in termini di attivazione (e non di mera mediazione) di pratiche, processi e attori, consente di cogliere il tratto 'generativo' che contraddistingue gli oggetti, le tecnologie e le architetture nei TDE. Al contempo, la presenza attiva di un ricercatore (e gli artefatti a ciò connessi) interpella di continuo gli attori circa le forme appropriate di uso di tali artefatti e di posizionamento organizzativo, fornendo loro 
un'occasione per esplicitare le reciproche aspettative e problematizzare in senso costruttivo le pratiche organizzative quotidiane.

Con questo numero speciale abbiamo intesto dunque riportare Studi Organizzativi a un tema classico, quale il rapporto tra tecnologia e organizzazione. In accordo con la recente letteratura al crocevia tra Science and Technolgy Studies e studi organizzativi, proponiamo uno spostamento d'attenzione dalla tecnologia in sé alla consistenza materiale dell'agire organizzativo. Si tratta di uno spostamento reso possibile dalla curvatura del dibattito, che assume oggi una prospettiva relazionale e sempre più orientata agli usi situati degli artefatti tecnologici.

In tale contesto ci sembra che il costrutto di "ambiente tecnologicamente denso" sia capace di indicare efficacemente direzioni di ricerca nelle quali appare possibile rintracciare lo snodarsi dei processi costitutivi dell'organizzare. Gli articoli che contribuiscono a questo numero speciale ci permettono di mostrare come rendere conto del lavorio quotidiano che i diversi attori (umani e non umani) sono chiamati a svolgere per assicurare coerenza e consistenza all'organizzazione.

Lungi dal rappresentare uno specialismo connesso alla particolarità di organizzazioni complesse e ad alta intensità tecnologica, tale costrutto permette invece di concentrare l'attenzione sul lavoro invisibile di tecnologie, oggetti, infrastrutture, attori e network organizzativi e di portare alla luce le complesse pratiche di coordinamento e di orchestrazione delle conoscenze tacite connesse alle pratiche esperte che prendono forma in ogni ambiente organizzativo.

Convinti che l'attenzione alla consistenza materiale della quotidianità organizzativa possa offrire l'occasione di avvicinare ancora di più la ricerca organizzativa alla comprensione dei processi costitutivi del fare organizzazione oggi, lasciamo la parola agli autori e alle autrici che hanno contribuito a questo numero.

\section{Bibliografia di riferimento}

Agar, M. (1980), The Professional Stranger, New York, Academic Press.

Albolino, S., Cook, R. (2005), "Medici in terapia intensiva: sensemaking, sicurezza e lavoro quotidiano”, Studi organizzativi, 2: 7-28.

Alby, F., Zucchermaglio, C. (2006), “Afterwards we can understand what went wrong, but now let's fix it: How Situated Work Practices Shape Group Decision Making in Organizations”, Organization Studies, 7: 943-966. 
Amalberti, R., Auroy Y., Berwick, D., Barach, P. (2005), "Five System Barriers to Achieving Ultrasafe Health Care”, Annals of Internal Medicine, 9: 756764.

Anderson, R.A., McDaniel, R.R. (2000), "Managing Healthcare Organizations: Where Professionalism Meets Complexity Science”, Health Care Manage Review, 1:83-92.

Ball, M.J., Weaver, C. Abbott, P. (2003), "Enabling Technologies Promise to Revitalize the Role of Nursing in an Area of Patient Safety”, International Journal of Medical Informatics, 1: 29-38.

Barley, S., Kunda, G. (2001), “Bringing Work Back In”, Organization Science, 1: 76-95.

Beunza, D., Stark, D. (2003), "Tools of the Trade: The Socio-technology of Arbitrage in a Wall Street Trading Room”, Industrial and Corporate Change, 13: 369-400.

Blumer, H. (1969), Symbolic Interactionism: Perspective and Method, New Jersey, Prentice-Hall.

Bruni, A. (2010), La sicurezza organizzativa. Una etnografia in sala operatoria, Roma, Carocci.

Bruni, A. (2003), Lo studio etnografico delle organizzazioni, Roma, Carocci.

Bruni, A. (2005a), "Shadowing Software and Clinical Records: On the Ethnography of Non-Humans and Heterogeneous Contexts", Organization, 12: 357-78.

Bruni, A. (2005b), "La socialità degli oggetti e la materialità dell’organizzare: umani e non-umani nei contesti lavorativi", Studi Organizzativi, 1: 113129.

Bruni, A. (2006), "Have You Got a Boyfriend or are You Single?: On the Importance of Being 'Straight' in Organizational Research”, Gender, Work and Organization, 3: 299-316.

Bruni, A. (2007), "Access as Trajectory: Entering the Field in Organizational Ethnography”,M@n@gement, 3: 129-144.

Bruni, A. (2008), "La medicina come ingegneria dell'eterogeneo e pratica sociomateriale”, Rassegna Italiana di Sociologia, 3: 451-476.

Bruni, A., Gherardi, S. (2007), Studiare le pratiche lavorative, Bologna, Il Mulino.

Bruni, A., Modé, D. (2011), 7+2: studiare le trame del lavoro in centrale operativa, Tecnoscienza, Italian Journal of Science\&Technology Studies, 2, 2:3-27.

Bruni, A., Viteritti, A. (2011), "Etnografia, persone e cose”, in Krippendorff, K., La Rocca, G. (eds.), Ricerca qualitativa e giovani studiosi. Atti del Convegno Internazionale RiQGioS-2011, Social Books, Palermo: 51-56. 
Bruni, A., Pinch, T., Schubert, C. (2013), “Technologically Dense Environments: What For? What Next?”, Tecnoscienza. Italian Journal of Science\&Technology Studies, 4: 51-72.

Butera, F. (2009), Il cambiamento organizzativo. Analisi e progettazione, Laterza.

Cardano, M. (2003), Tecniche di ricerca qualitativa. Percorsi di ricerca nelle scienze sociali, Roma, Carocci.

Casper, M.J., Berg, M. (1995), “Constructivistic Perspectives on Medical Work: Medical Practices and Science and Technology Studies”, Science, Technology, and Human Values, 20: 395-407.

Cedefop, (2010), New Skills for New Jobs: Action Now, Report by the Expert Group on New Skills for New Jobs, prepared for the European Commission. Cooper, R., Law, J. (1995), “Distal and Proximal Visions of Organization”, in Bacharach, S., Gagliardi, P., Mundell, B. (eds), Studies of Organizations in the European Tradition, Greenwich, Jai Press (trad. it. "Visioni distali e prossimali dell’organizzazione”, in Bacharach S., Gagliardi, P., Mundell, B. (eds.), Il pensiero organizzativo europeo, Milano, Guerini e Associati).

Corbin, J., Strauss, A. (1993), “The Articulation of Work Through Interaction”, Sociological Quarterly, 1: 71-83.

Czarniawska, B., Hernes, T. (eds.), (2005), Actor-Network Theory and Organizing, Copenhagen, Liber \& Copenhagen Business School Press.

Danholt, P., Piras, E.M., Storni, C. Zanutto, A. (2013), “The shaping of patient 2.0.: exploring agencies, technologies and discourses in new healthcare practices”, Science \& Technology Studies, 3-13.

Emery, F.E., Trist, E.L. (1993), “The causal texture of organizational environments”, in Trist, E.L., Murray, H. (eds.), The Social Engagement of Social Science, Vol. II, Philadelphia, University of Pennsylvania Press.

Engeström, Y., Middleton D., (eds) (1996), Cognition and Communication at Work, Cambridge, Cambridge University Press.

Gherardi, S., Lippi, A., (eds.), (2000), Tradurre le riforme in pratica, Milano, Cortina.

Gherardi, S., Strati, A. (eds.), (2004), Telemedicina: tra tecnologia $e$ organizzazione, Roma, Carocci.

Gherardi, S., Lippi, A. (eds.), (2000), Tradurre le riforme in pratica, Milano, Raffaello Cortina Editore.

Gibson, J. J. (1979), The ecological approach to visual perception, New York, Houghton Mifflin.

Goffman, E. (1959), The Presentation of Self in Everyday Life, Garden City, NY, Doubleday (trad. it. La vita quotidiana come rappresentazione, Bologna, Il Mulino, 1979). 
Gursteun, B. (2011) Open data: Empowering the empowered or effective data use for everyone?, First Monday, 16, 2, doi:10.5210/FM.V16I2.3316.

Harré, R. (2002), Cognitive Science: A Philosophical Introduction, London, Sage.

Heath, C., Knoblauch, H., Luff, P. (2000), “Technology and Social Interaction: the emergence of workplace studies”, British Journal of Sociology, 51: 299-320, doi: 10.1111/J.1468-4446.2000.00299.X.

Heath, C., Luff, P. (2000), Technology in Action, Cambridge, Cambridge University Press,.

Hughes, E.C. (1958), Men and their Work, Glencoe, The Free Press.

Ingold, T. (2000), The perception of the environment: essays on livelihood, dwelling and skill, London, Routledge.

Knorr-Cetina, K. Preda. A. (eds.), (2005) The Sociology of Financial Markets. Oxford University Press.

Knorr-Cetina, K. (1997), "Sociality with Objects”, Theory, Culture and Society, 14: 1-30, doi: 10.1177/026327697014004001.

Lash, S. (2001), “Technological Forms of Life”, Theory, Culture and Society, 18: 105-120, doi: 10.1177/02632760122051661.

Latour, B. (2005), Reassembling the Social. An Introduction to Actor-NetworkTheory, Oxford, Oxford University Press.

Latour, B., Woolgar, S. (1986), Laboratory life: the construction of scientific facts, Princeton, Princeton University.

Lave, J., Wenger, E. (1991), Situated Learning. Legitimate Peripheral Participation, Mass, Cambridge, Cambridge University Press (trad. it. L'apprendimento situato. Dall'osservazione alla partecipazione attiva nei contesti sociali, Trento, Erickson, 2006).

Lave, J. (1988), Cognition in practice: Mind, mathematics and culture in everyday life, New York, Cambridge University Press.

Law, J. (1994), Organizing Modernity, Oxford, Blackwell.

Luff, P., Hindmarsh, J., Heath, C. (eds.), (2000), Workplace Studies: Recovering Work Practice and Informing System Design, Cambridge, Cambridge University Press.

Lynch, M., Collins, H. M. (eds.), (1998), Humans, Animals and Machines: Special edition of Science Technology and Human Values, 23, 4: 371-490, Beverley Hills: Sage.

Mantovani, G. (2004), Intercultura, Bologna, Il Mulino.

March, J.G. (1988), Decisions and Organizations, New York, Basil Blackwell (trad. it. Decisioni e organizzazioni, Bologna, Il Mulino, 1993).

Mattozzi, A. (2006), (eds.), Il senso degli oggetti tecnici, Roma, Meltemi. 
Morgan, G. (1986), Images of Organization, Beverly Hills, CA, Sage (trad. it. Images. Le metafore dell'organizzazione, Milano, Franco Angeli, 1989).

Mort, M., May, C., Williams, T. (2003), "Remote Doctors and Absent Patients: Acting at a Distance in Telemedicine”, Science, Technology and Human Values, 28: 274-295.

Orlikowski, W.J., Scott, S.V. (2008), "Sociomateriality: Challenging the separation of technology, work and organization”, Academy of Management Annals, 2: 433-474, doi: 10.1080/19416520802211644.

Orr, J. (1996), Talking about machines: an ethnography of a modern job, Ithaca, N.Y., ILR Press.

Parolin, L. (2011), Tecnologia e sapere pratico nella società della conoscenza, Milano, Franco Angeli.

Perrow, C. (1984), Normal Accidents: Living with High-Risk Technologies, New York, Basic Books.

Piccardo, C., Benozzo, A. (1996), Etnografia organizzativa: una proposta di metodo per l'analisi delle organizzazioni come culture, Milano, Cortina.

Pinch, T. Swedberg, R. (2008), Living in a Material World: Economic Sociology Meets Science and Technology Studies, vol. 1, Ed., The MIT Press.

Rifkin, J. (1995), The end of work: The decline of the global labour force and the dawn of postmarket era, New York, Putnam.

Schutz, A., (1932), Der Sininnhafte Aufbau der sozialen Welt, Wien, Springer (trad. it. La fenomenologia del mondo sociale, Bologna, Il Mulino, 1974).

Silverman, D. (1997), Doing Qualitative Research, Sage, London (trad. it. Come fare ricerca qualitativa, Roma, Carocci, 2002).

Star, S.L., Strauss, A. (1998), "Layers of Silence, Arenas of Voice: The Ecology of Visible and Invisible Work”, Computer Supported Cooperative Work, 8: 9-30.

Suchman, L. (2002), "Practice-based design of information systems: notes from the hyperdeveloped world”, The Information Society, 18 (2):139-144.

Suchman, L. (1987), Plans and Situated Action: The Problem of Humanmachine Communication, Cambridge, Cambridge University Press.

Suchman, L. (1997), “Centers of Coordination. A Case and Some Themes”, in Resnik, L., Saljo, L., Pontecorvo, C., Burge, B., (eds.), Discourse, Tools and Reasoning. Essays On Situated Cognition, Berlin, Springer-Verlag.

Suchman, L., Blomberg, J., Orr, J.E., Trigg, R. (1999), "Reconstructing Technology as Social Practice”, American Behavioral Scientist, 43: 392408.

Suchman, L. (2000), “Organizing Alignment: A Case of Bridge-Building”, Organization, 7: 311-327, doi: 10.1177/135050840072007. 
Suchman, L. (2007), Human-Machine Reconfigurations: Plans and situated actions (second edition), Cambridge, Cambridge University Press.

Timmermans, S. (2000), "Technology and Medical Practice", in Bird, C., Conrad, P., Fremont, A., (eds.), Handbook of Medical Sociology $\left(5^{\circ}\right.$ edizione), Upper Saddle River, New Jersey, Prantice Hall, Inc.

Viteritti, A. (2012), Scienza in Formazione. Corpi, materialità e scrittura in laboratorio, Milano, Guerini Scientifica.

Vygotskij, L.S., (1934), Pensiero e linguaggio, Roma- Bari, Laterza.

Weick, K. E. (1979), The Social Psichology of Organizing, Reading, MA, Addison-Wesley (trad. it. Organizzare, La psicologia sociale dei processi organizzativi, Torino, ISEDI, 1993).

Weick, K.E. (1990), "Technology as Equivoque: Sensemaking in New Technologies”, in Goodman, P., Sproull, L.S. (eds.), Technology and Organizations, San Francisco, Jossey Bass.

Whalen, J., Whalen, M., Henderson, K. (2002), "Improvisational Coreography in Teleservice Work”, British Journal of Sociology, 53: 239-258.

Wiener, N. (1950), The human use of human beings: Cybernetics and society, New York, Da Capo Press.

Woodward, J. (1965), Industrial organization: theory and practice, London, Oxford University Press.

Zuboff, S. (1988), In The Age of the Smart Machine: The Future of Work and Power, New York, Basic Books.

Zucchermaglio, C., Alby, F., Fatigante, M., Saglietti, M. (2013), Fare ricerca situata in psicologia sociale, Bologna, Il Mulino. 